\title{
Accurate Measurement of Arsenic in Laver by Gravimetric Standard Addition Method Combined with High Resolution Inductively Coupled Plasma Mass Spectrometry
}

\author{
Kyoung-Seok Lee ${ }^{1 *}$, Hyeon-Ji Kim ${ }^{1,2}$, Yong-Hyeon Yim ${ }^{1}$, Jeongkwon Kim ${ }^{2}$, and Euijin Hwang ${ }^{1}$ \\ ${ }^{1}$ Korea Research Institute of Standards and Science (KRISS), Daejeon 305-340, South Korea \\ ${ }^{2}$ Department of Chemistry, Chungnam National University, Deajeon 305-764, South Korea
}

Received June 03, 2014; Revised June 10, 2014; Accepted June 11, 2014

First published on the web June 30, 2014; DOI: 10.5478/MSL.2014.5.2.57

\begin{abstract}
A gravimetric standard addition method combined with internal standard calibration has been successfully developed for the accurate analysis of total arsenic in a laver candidate reference material. A model equation for the gravimetric standard addition approach using an internal standard was derived to determine arsenic content in samples. Handlings of samples, As standard and internal standard were carried out gravimetrically to avoid larger uncertainty and variability involved in the volumetric preparation. Germanium was selected as the internal standard because of its close mass to the arsenic to minimize mass-dependent bias in mass spectrometer. The ion signal ratios of ${ }^{75} \mathrm{As}^{+}$to ${ }^{72} \mathrm{Ge}^{+}$(or ${ }^{73} \mathrm{Ge}^{+}$) were measured in high resolution mode (R $\geq 10,000$ ) to separate potential isobaric interferences by high resolution ICP/MS. For method validation, the developed method was applied to the analysis of arsenic content in the NMIJ 7402-a codfish certified reference material (CRM) and the result was $37.07 \mathrm{mg} \cdot \mathrm{kg}$ ${ }^{1} \pm 0.45 \mathrm{mg} \cdot \mathrm{kg}^{-1}$ which is in good agreement with the certified value, $36.7 \mathrm{mg} \cdot \mathrm{kg}^{-1} \pm 1.8 \mathrm{mg} \cdot \mathrm{kg}^{-1}$. Finally, the certified value of the total arsenic in the candidate laver CRM was determined to be $47.15 \mathrm{mg} \cdot \mathrm{kg}^{-1} \pm 1.32 \mathrm{mg} \cdot \mathrm{kg}^{-1}$ ( $k=2.8$ for $95 \%$ confidence level) which is an excellent result for arsenic measurement with only $2.8 \%$ of relative expanded uncertainty.
\end{abstract}

Keywords : arsenic, gravimetric standard addition, internal standard, laver, certified reference material

\section{Introduction}

Many countries set regulations on the concentrations of arsenic in drinking water and foods due to its well-known toxicity. ${ }^{1-4}$ Elevated dietary exposure to high levels of arsenic can be a potential threat to human health. Especially in sea weeds, arsenic has been frequently found in concentrations exceeding the regulation limits. In particular, laver has been traditionally consumed in large quantities in far-east Asian countries and, nowadays, they are gaining widespread popularity worldwide. Therefore, the accurate determination of arsenic in laver can be a matter of concern for the food safety in many counties. ${ }^{2-5}$ In order to assure the quality of measurement results, use of relevant certified reference

\section{Open Access}

*Reprint requests to Kyoung-Seok Lee

E-mail: kslee@kriss.re.kr

All MS Letters content is Open Access, meaning it is accessible online to everyone, without fee and authors' permission. All MS Letters content is published and distributed under the terms of the Creative Commons Attribution License (http://creativecommons.org /licenses/by/3.0/). Under this license, authors reserve the copyright for their content; however, they permit anyone to unrestrictedly use, distribute, and reproduce the content in any medium as far as the original authors and source are cited. For any reuse, redistribution, or reproduction of a work, users must clarify the license terms under which the work was produced. material (CRM) has been recommended to analytical laboratories for the method validation and quality control in ISO/IEC $17025 .^{6}$ The development of CRM for arsenic analysis in laver is also very important as a means to assist field laboratories to get reliable measurement results.

Accurate determination of elemental contents in a complex matrix is best realized using primary methods with the highest level of reliability including the isotope dilution (ID) inductively-coupled plasma mass spectrometry (ICP/MS). However, the ID ICP/MS analysis of arsenic is impossible because arsenic has only one stable isotope. Application of another potential primary method, the instrumental neutron activation analysis (INAA), is often not practically possible due to limited accessibility to facilities with neutron radiation source. ${ }^{7}$ As an alternative approach, development of an arsenic measurement method based on conventional analytical methods such as ICP/MS can be also considered provided that rigorous measurement and calibration strategies are implemented.

For the accurate determination of arsenic using ICP/MS, it is essential to exclude spectral interferences to ${ }^{75} \mathrm{As}^{+}$from isobars such as ${ }^{40} \mathrm{Ar}^{35} \mathrm{Cl}^{+}$and ${ }^{59} \mathrm{Co}^{16} \mathrm{O}^{+}$. For this purpose, either higher mass resolution ${ }^{8}$ or collision/reaction cells are used. Non-spectral matrix effect is another important factor to be controlled to get right results from the ICP/MS measurement. Arsenic can be separated from the sample 
matrix to remove the matrix effect but incomplete recovery of arsenic and lack of robust recovery correction method poses a significant challenge to develop it as a reliable analysis method. Standard addition method (SAM) is one of the robust calibration method for ICP/MS analysis to overcome the measurement bias stemming from matrix effects without matrix separation. ${ }^{9,10}$ Provided that the procedure blank is tightly controlled and evaluated, it is expected to produce reliable result even in the presence of severe matrix effects. One of the pitfalls of the SAM, however, is relatively large uncertainty as a natural consequence of the extrapolation calibration. In order to achieve the highest level of accuracy to the extent of assigning the certified value for CRM development, gravimetric sample handling in the standard addition procedure is necessary as well as the use of appropriate internal standards. ${ }^{11}$ In this study, an ICP/MSbased arsenic measurement procedure was established with rigorous gravimetric standard addition method (SAM) combined with internal standard calibration as the most robust calibration strategy next to the ID ICP/MS. The method was applied to measure arsenic content in NMIJ 7402-a codfish CRM for method validation and certification of arsenic content laver candidate CRM.

\section{Experimental}

\section{Materials and reagents}

The dry laver powder sample was provided by the National Institute of Metrology (NIM), China, which is a candidate reference material of the Asian Collaboration on Reference Materials (ACRM) program. The codfish CRM (NMIJ CRM 7402-a) used for method validation and quality control was obtained from the National Metrology Institute of Japan (NMIJ). The arsenic standard solution for standard additions and the germanium standard solution for internal standard were produced by Korea Research Institute of Standards and Science (KRISS) with traceability to the SI unit. The working standard solutions of the arsenic and the germanium were freshly prepared just before sample preparation by diluting the stock solutions of $1000 \mathrm{mg} \cdot \mathrm{kg}^{-1}$ with $5 \%$ (w/w) nitric acid. Nitric acids with various concentrations used throughout this study were prepared just before sample preparation by diluting the high purity concentrated nitric acid $(\geq 65 \%)$ with deionized water (18 MW $\cdot \mathrm{cm}$ resistivity) from Milli-Q RG purification system (Millipore, USA). The high purity concentrated nitric acid was obtained by sub-boiling distillation of commercially available concentrated nitric acid from DONGWOO FINECHEM (Iksan, Korea).

\section{Sample preparation with standard addition procedure}

About $0.2 \mathrm{~g}$ of laver powder from each bottle was sampled and weighed directly into a PTFE-TFM microwave vessel. Total six subsamples from different bottles of laver powder were prepared. Then, about $1 \mathrm{~g}$ of the germanium working standard solution with an appropriate mass fraction was added in each PTFE-TFM vessel and weighed exactly. For standard addition calibration, different amounts of the arsenic working standard solution were added in five levels from $1 \mathrm{~g}$ to $5 \mathrm{~g}$, respectively, into the five PTFE-TFM vessels containing both sample and internal standard. The remaining one vessel was prepared without adding the arsenic working standard solution. Then, the samples in the six vessels were digested with $8 \mathrm{~mL}$ of concentrated nitric acid and $2 \mathrm{~mL}$ of $30 \%$ hydrogen peroxide solution using microwave digestion system (ETHOS PLUS, Milestone, USA). Microwave-assisted acid digestion was carried out with the temperature control program to increase temperature from room temperature to $200^{\circ} \mathrm{C}$ for $10 \mathrm{~min}$ and maintain it for $10 \mathrm{~min}$. The digested samples were transferred into LDPE bottles and diluted appropriately for ICP/MS measurements. The analysis of the NMIJ 7402-a codfish CRM was also carried out for the validation of the developed method. Six aliquots ( $0.2 \mathrm{~g}$ each) of codfish powder from a single bottle were sampled individually in six PTFETFM microwave vessels. Then, the same procedure was used for preparation of standard addition samples.

For dry mass correction of laver powder, three subsamples were taken in parallel with the sampling for arsenic analysis. The subsample was about $0.2 \mathrm{~g}$ each and accurately weighed in a weighing bottle. After the samples in weighing bottles were dried at $102^{\circ} \mathrm{C}$ for 12 hours using a drying oven, the dried weights of the subsamples were measured. Finally, the losses of weights were used for the dry mass correction. In the dry mass correction for NMIJ 7402-a codfish sample, the procedure was the same except for taking $0.3 \mathrm{~g}$ of subsamples and drying at $102^{\circ} \mathrm{C}$ for 6 hours following the instruction of the certificate.

\section{High resolution (HR) ICP/MS measurements of arsenic and germanium}

Signal intensities as counts per second (cps) of arsenic ions $\left({ }^{75} \mathrm{As}^{+}\right)$and germanium ions $\left({ }^{72} \mathrm{Ge}^{+}\right.$and $\left.{ }^{73} \mathrm{Ge}^{+}\right)$were measured using an HR ICP/MS (Element2, Thermo Fisher Scientific Inc., Germany) at high resolution mode. Sample solution was nebulized through quartz micro-concentric nebulizer at a flow rate of $400 \mathrm{~mL} \cdot \mathrm{min}^{-1}$ into the Scott-type quartz spray chamber using a peristaltic pump. Fine aerosols generated in the spray chamber were introduced into the ICP plasma by quartz torch and injector. The operating conditions of HR ICP/MS are summarized in Table 1.

Table 1. Optimized operating conditions of HR ICP/MS for measurements of arsenic and germanium

\begin{tabular}{cc}
\hline \hline Measured ions & ${ }^{72} \mathrm{Ge}^{+},{ }^{73} \mathrm{Ge}^{+},{ }^{75} \mathrm{As}^{+}$ \\
Sampler/skimmer cones & $\mathrm{Ni}$ \\
Plasma gas flow rate & $16 \mathrm{~L} \cdot \mathrm{min}^{-1}$ \\
Auxiliary gas flow rate & $0.8 \mathrm{~L} \cdot \mathrm{min}^{-1}$ \\
Nebulizer gas flow rate & $0.934 \mathrm{~L} \cdot \mathrm{min}^{-1}$ \\
Plasma RF power & $1250 \mathrm{~W}$ \\
Resolution & High resolution $(\mathrm{R} \sim 10,000)$ \\
\hline
\end{tabular}




\section{Results and Discussion}

\section{Gravimetric SAM with internal standard: Measurement Equation}

The model equation for the gravimetric SAM for the analysis of the element, E, with the internal standard, IS, was derived for the analysis of total arsenic by ICP/MS. In this method, all the handlings of sample $\mathrm{X}$, internal standard solution $\mathrm{Y}$, and standard solution $\mathrm{Z}$ are carried out gravimetrically as shown by Figure 1 . For an $i^{\text {th }}$ solution, mass $m_{Z, i}$ of standard $\mathrm{Z}$ with mass fraction $w_{\mathrm{Z}}(\mathrm{E})$ is added into the mixture consisted of mass $m_{\mathrm{X}, i}$ of sample $\mathrm{X}$ with mass fraction $w_{\mathrm{X}}(\mathrm{E})$ and mass $m_{\mathrm{Y}, i}$ of internal standard $\mathrm{Y}$ with mass fraction $w_{\mathrm{Y}}$ (IS). Then, ICP/MS measures the ion intensities of $\mathrm{E}$ and IS, $R_{i}(\mathrm{E})$ and $R_{i}(\mathrm{IS})$ which are proportional to the number of atoms of $\mathrm{E}$ and IS, $N_{i}(\mathrm{E})$ and $N_{i}(\mathrm{IS})$, respectively.

$$
R(\mathrm{E})=k_{i}(\mathrm{E}) \cdot N_{i}(\mathrm{E}), R_{i}(\mathrm{IS})=k_{i}(\mathrm{IS}) \cdot N_{i}(\mathrm{IS})
$$

where $k_{i}(\mathrm{E})$ and $k_{i}(\mathrm{IS})$ represent ICP/MS sensitivities of $\mathrm{E}$ and IS in the $i^{\text {th }}$ sample. The ratio of responses can be written as follows:

$$
\begin{aligned}
& R_{i}=\frac{R_{i}(E)}{R_{i}(I S)}=\frac{k_{i}(E)}{k_{i}(I S)} \cdot \frac{N_{i}(E)}{N_{i}(I S)} \\
& =\frac{k_{i}(E)}{k_{i}(I S)} \cdot \frac{\left\{\frac{m_{X, i} \cdot w_{X}(E)+m_{Z, i} \cdot w_{Z}(E)}{M(E)}\right\}}{\left\{\frac{m_{Y, i} \cdot w_{y}(I S)}{M(I S)}\right\}},
\end{aligned}
$$

where $M(\mathrm{E})$ and $M(\mathrm{IS})$ are atomic weights of $\mathrm{E}$ and IS, respectively. For simpler formula, new sensitivity factor, $k_{i}$, is redefined as follows,

$$
k_{i}=\frac{k_{i}(E)}{k_{i}(I S)} \cdot \frac{M(I S)}{M(E)} .
$$

Then,

$$
R=k_{i} \cdot \frac{m_{X, i} \cdot w_{X}(E)+m_{Z, i} \cdot w_{z}(E)}{m_{Y, i} \cdot w_{Y}(I S)} .
$$
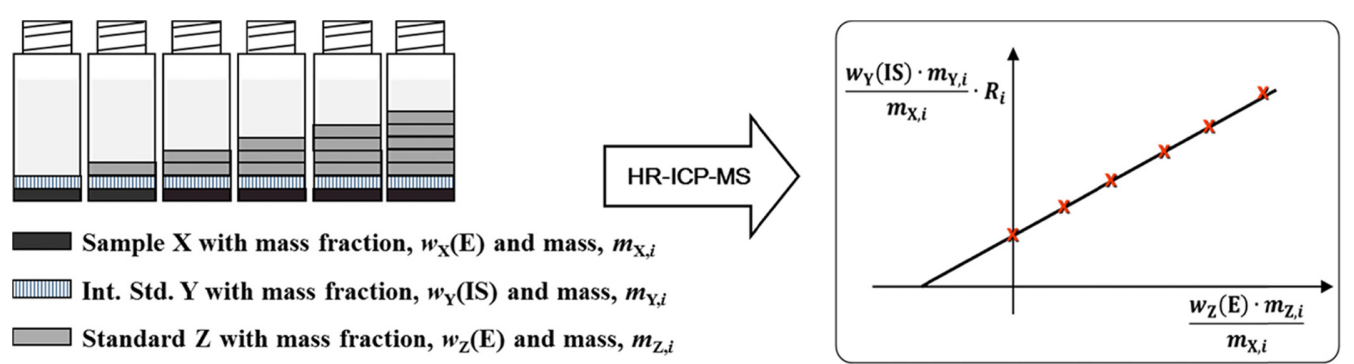

Figure 1. Schematic diagram of the gravimetric standard addition method combined with internal standard and HR ICP/MS. $R_{\mathrm{i}}$ represents the ratio of ion intensities of arsenic over germanium in each standard addition sample. All abbreviations are shown in theoretical section. 


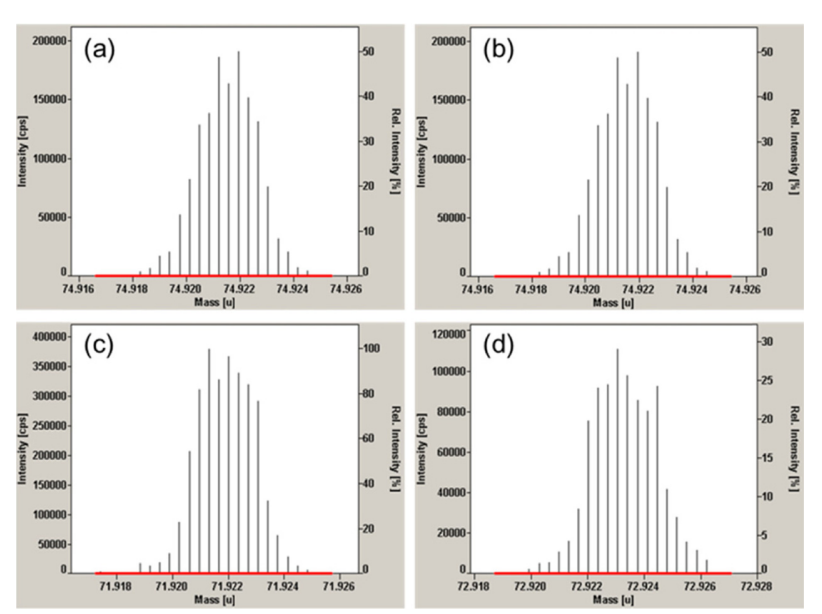

Figure 2. Typical HR ICP/MS mass spectra of (a) ${ }^{75} \mathrm{As}^{+}$ions from a digested sample, (b) ${ }^{75} \mathrm{As}^{+}$ions from ca. $20 \mu \mathrm{g} \cdot \mathrm{kg}^{-1}$ arsenic standard solution, (c) ${ }^{72} \mathrm{Ge}^{+}$ions and (d) ${ }^{73} \mathrm{Ge}^{+}$ions from a digested sample spiked with internal standard.

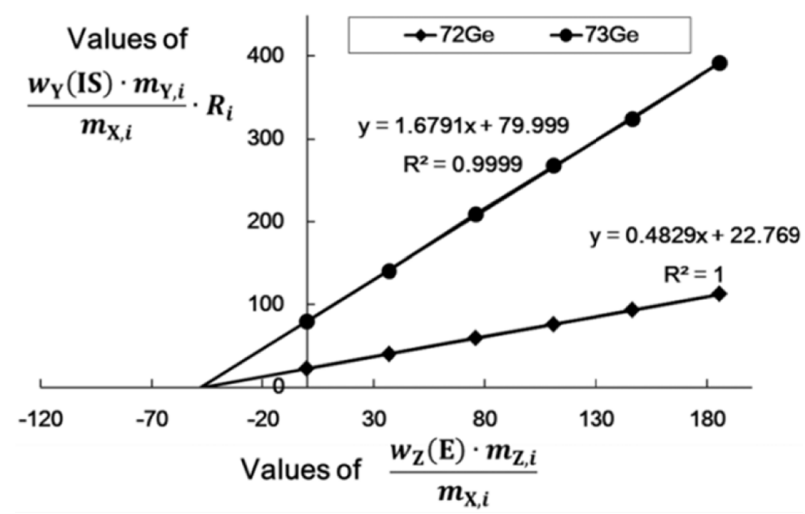

Figure 3. Standard addition calibration curves obtained for the HR ICP/MS-based arsenic measurement in laver samples using the model equation for the gravimetric standard addition method with germanium internal standard. $72 \mathrm{Ge}$ and $73 \mathrm{Ge}$ denote the experimental values for the ion signal ratios of ${ }^{75} \mathrm{As}^{+} /$ ${ }^{72} \mathrm{Ge}^{+}$and ${ }^{75} \mathrm{As}^{+} /{ }^{73} \mathrm{Ge}^{+}$, respectively.

resolution in the SF ICP/MS.

Standard addition calibration curves for determination of arsenic in laver samples using two different Ge isotopes, ${ }^{72} \mathrm{Ge}$ and ${ }^{73} \mathrm{Ge}$, as internal standards are shown in Figure 3. From the calibration curve, mass fraction of arsenic was calculated. Both of the two calibration curves show excellent linearity with regression coefficient of 1.0000 and 0.9999 , respectively, for ${ }^{72} \mathrm{Ge}$ and ${ }^{73} \mathrm{Ge}$ as internal standards. In the standard addition calibration, the ion signal ratios of ${ }^{75} \mathrm{As}^{+} /$ ${ }^{72} \mathrm{Ge}^{+}$were in a narrow range of 0.5 to 1.4 (from 1.7 to 4.7 for $\left.{ }^{75} \mathrm{As}^{+} /{ }^{73} \mathrm{Ge}^{+}\right)$to make the linear regression sufficiently accurate approximation. It should be noted that the 6 data used for linear regression include variations due to betweenbottle homogeneity. Although there was no noticeable difference between the two results obtained using the two different $\mathrm{Ge}$ isotopes as internal standards within the associated uncertainties, the result obtained using ${ }^{72} \mathrm{Ge}$ as an internal standard was finally used for the analysis because of its smaller uncertainty. Coincidence of the two results also provides a supporting evidence on the robustness of the present method for arsenic determination. In the case of NMIJ 7402-a codfish CRM, which was used for method validation, total arsenic content was measured to be $37.07 \mathrm{mg} \cdot \mathrm{kg}^{-1} \pm 0.45 \mathrm{mg} \cdot \mathrm{kg}^{-1}$ which is in good agreement with the certified value, $36.7 \mathrm{mg} \cdot \mathrm{kg}^{-1} \pm 1.8 \mathrm{mg} \cdot \mathrm{kg}^{-1}$. The certified value of the total arsenic in the candidate laver CRM was determined to be $47.15 \mathrm{mg} \cdot \mathrm{kg}^{-1} \pm 1.32 \mathrm{mg} \cdot \mathrm{kg}^{-1}(\mathrm{k}=2.8$ for $95 \%$ confidence level) which is an excellent result obtained by SAM with only $2.8 \%$ of relative expanded uncertainty including the homogeneity between bottles. The uncertainties of determined value were calculated following the ISO Guide to the Expression of Uncertainty in Measurement (GUM) guidelines ${ }^{12}$.

\section{Conclusion}

A gravimetric standard addition method combined with internal standard and HR ICP/MS has been successfully developed for the accurate determination of total arsenic content in laver candidate CRM. A measurement model equation of gravimetric SAM with internal standard was derived. Standard addition calibration curves obtained for gravimetric SAM using Ge internal standard showed excellent linearity, which results in relatively small measurement uncertainties even though they include contributions from between-bottle homogeneity. The developed method was validated using NMIJ codfish CRM and applied for the certification of KRISS laver CRM. The present strategy is expected to provide an alternative means to certify elemental content in complex matrix, when the other primary methods of analysis, such as ID ICP/MS and INAA, are not available.

\section{Acknowledgments}

The authors would like to thank Mr. Wei Chao and NIM for providing samples. This work was supported by the Korea Research Institute of Standards and Science (KRISS) under the project "Establishing Measurement Standards for Inorganic Analysis”, grant 14011010.

\section{References}

1. Mandal, B. K.; Suzuki, K. T. Talanta 2002, 58, 201.

2. Bhattacharya, P.; Welch, A. H.; Stollenwerk, K. G.; McLaughlin, M. J.; Bundschuh, J.; Panaullah, G. Sci. Total Environm. 2007, 379, 109.

3. World Health Organization (WHO) Environmental health criteria 224, arsenic and arsenic compounds. Interorganization programme for the sound management of 
chemials. Generva; 2001.

4. Rose, M.; Lewis, J.; Langford, N.; Baxter, M.; Origgi, S.; Barber, M.; MacBain, H.; Thomas, K. Food Chem. Toxicol. 2007, 45, 1263-1267.

5. Melamed, D. Anal. Chim. Acta 2005, 532, 1.

6. ISO/IEC 17025:2005 General requirements for the competence of testing and calibration laboratories, 2nd Ed.; ISO: Geneva, Switzerland, 2005.

7. Zeisler, R.; Mackey, E. A.; Lamaze, G. P.; Stover, T. E.; Sflaz Spatz, R.; Greenberg, R. R. J. Radioanal. Nucl. Chem. 2006, 269, 291.
8. Kohlmeyer, U.; Jantzen, E.; Kuballa, J.; Jakubik, S. Anal. Bioanal. Chem. 2003, 377, 6.

9. Saxberg, B. E. H.; Kowalski, B. R. Anal. Chem. 1979, 51, 1031.

10. Heitkemper, D. T.; Vela, N. P.; Stewart, K. R.; Westphal, C. S. J. Anal. At. Spectrom. 2001, 16, 299.

11. Hauswaldt, A. L.; Rienitz, O.; Jährling, R.; Fischer, N.; Schiel, D.; Labarraque, G.; Magnusson, B. Accred. Qual. Assur. 2012, 17, 129.

12. Guide to the Expression of Uncertainty in Measurement. ISO: Geneva, 1995. 